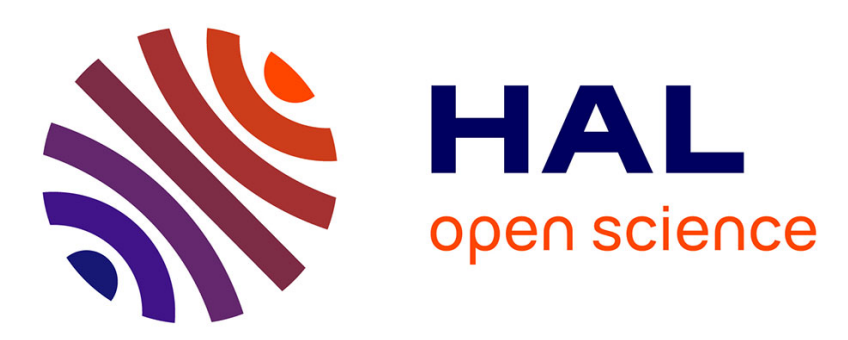

\title{
Transient Quasi-Static Gas Flow through a Rigid Porous Medium with Double Porosity
}

Pascale Royer, Jean-Louis Auriault

\section{To cite this version:}

Pascale Royer, Jean-Louis Auriault. Transient Quasi-Static Gas Flow through a Rigid Porous Medium with Double Porosity. Transport in Porous Media, 1994, 17 (1), pp.33-57. 10.1007/BF00624049 . hal-01713395

\section{HAL Id: hal-01713395 \\ https://hal.science/hal-01713395}

Submitted on 20 Feb 2018

HAL is a multi-disciplinary open access archive for the deposit and dissemination of scientific research documents, whether they are published or not. The documents may come from teaching and research institutions in France or abroad, or from public or private research centers.
L'archive ouverte pluridisciplinaire HAL, est destinée au dépôt et à la diffusion de documents scientifiques de niveau recherche, publiés ou non, émanant des établissements d'enseignement et de recherche français ou étrangers, des laboratoires publics ou privés. 


\title{
Transient Quasi-Static Gas Flow through a Rigid Porous Medium with Double Porosity
}

\author{
PASCALE ROYER and JEAN-LOUIS AURIAULT \\ Laboratoire 'Sols, Solides, Structures' Institut de Mécanique de Grenoble, Loboratoire \\ associé CNRS, BP 53X, 38041 Grenoble Cedex, France
}

\begin{abstract}
The highly compressible fluid flow through a three-scales rigid porous medium (pore, fracture, macroscopic sample) is investigated using a homogenization method. The macroscopic description is strongly dependent on the separation of the different scales, and three cases are considered. The pores either play the role of a compressible fluid reservoir, introduce a memory effect, or are ignored, respectively. The homogenization result is compared to classical phenomenological models that are available in the case of slightly compressible fluids. Pseudo-steady state models are shown to give a rough description of the phenomenon.
\end{abstract}

Key words: double porosity, fractured porous media, gas filtration, homogenization.

\section{Nomenclature}

\section{Roman Letters}

$$
\begin{aligned}
& C^{*} \quad=\text { gas compressibility coefficient. } \\
& \text { f } \quad=\text { subscript for the fractures. } \\
& \mathcal{F}=\text { nonlinear function. } \\
& h \quad=\text { positive integer. } \\
& \tilde{I} \quad=\text { identity tensor. } \\
& k \quad=\text { subscript taking on the values } \mathrm{p} \text { for the pores and } \mathrm{f} \text { for the } \\
& \text { fractures. } \\
& \tilde{k}_{\mathrm{p}}, \tilde{k}_{\mathrm{f}} \quad=\text { particular solutions for the velocity fields in the pores and the } \\
& \text { fractures, respectively. } \\
& \tilde{K}_{\mathrm{p}}, \tilde{K}_{\mathrm{f}} \quad=\text { pore and fracture permeability tensors, respectively. } \\
& l, l^{\prime}, l^{\prime \prime} \quad=\text { characteristic lengths for the pore scale, the fracture scale and } \\
& \text { the macroscopic medium, respectively. } \\
& m \quad=\text { positive integer. } \\
& n, n^{\prime} \quad=\text { pore porosity and fracture porosity, respectively. } \\
& \mathbf{n}^{\prime} \quad=\text { normal unit vector. } \\
& \mathrm{p} \quad=\text { subscript for the pores. } \\
& P_{0} \quad=\text { initial pressure. }
\end{aligned}
$$




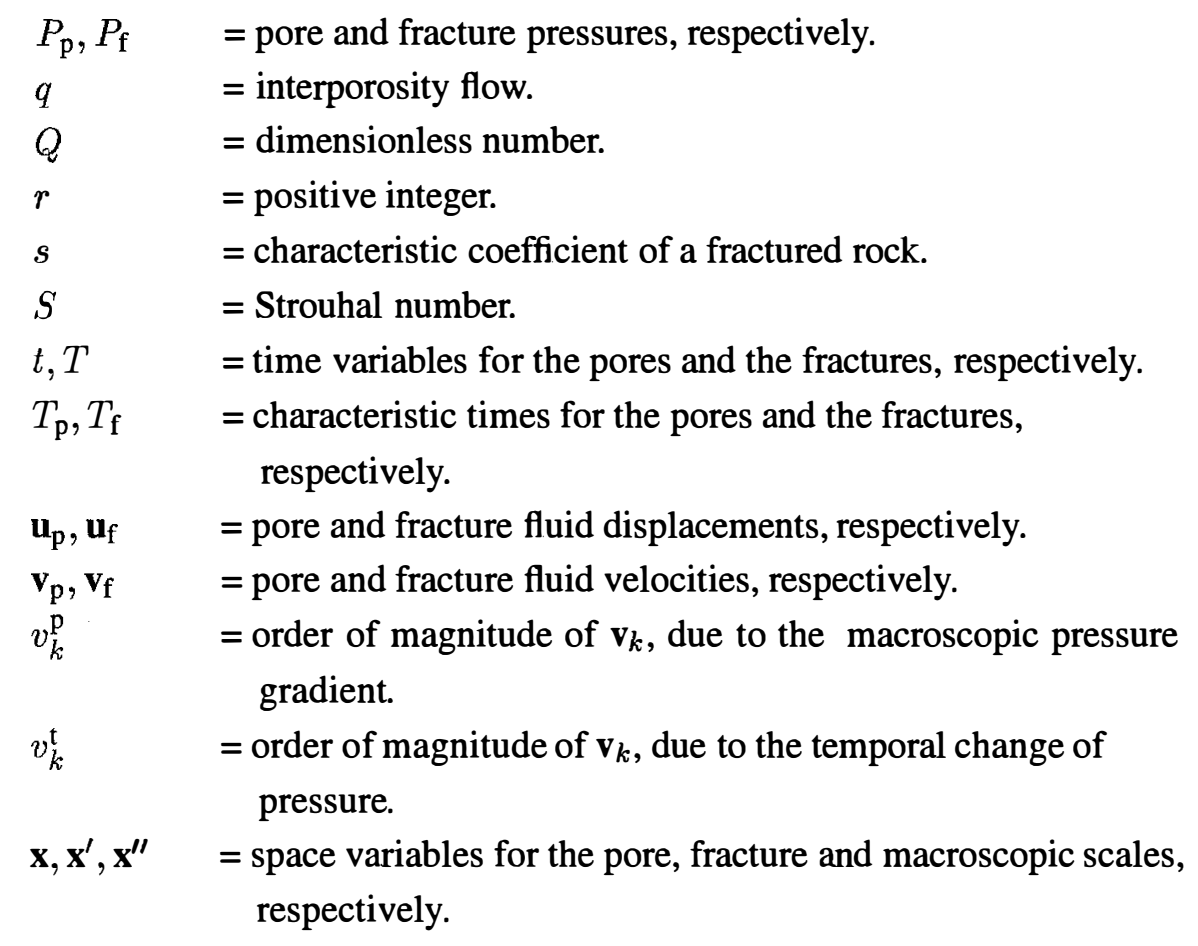

\section{Greek Letters}

$\alpha, \beta, \gamma \quad=$ ratios between the different characteristic lengths.

$\Gamma, \Gamma^{\prime} \quad=$ boundaries of the pores and the fractures, respectively.

$\Delta \quad=$ Laplace operator.

$\nabla \quad=$ gradient operator.

$\varepsilon \quad=$ small parameter.

$\lambda, \mu \quad=$ fluid viscosities.

$\rho_{0} \quad=$ initial density.

$\rho_{\mathrm{p}}, \rho_{\mathrm{f}} \quad=$ pore and fracture densities, respectively.

$\tau \quad=$ particular solution for the pressure.

$\tau_{\mathrm{p}}, \tau_{\mathrm{f}}=$ characteristic times for the pores and the fractures, respectively.

$\omega \quad=$ pulsation.

$\Omega, \Omega^{\prime} \quad=$ periods at the pore and fracture scales, respectively.

$\Omega_{\mathrm{p}}, \Omega_{\mathrm{sp}}^{\prime}, \Omega_{\mathrm{f}}^{\prime}=$ parts of the period occupied by the pores, the solid plus the pores and the fractures. 
Particular Symbols

$\langle\phi\rangle_{\Omega},\langle\phi\rangle_{\Omega^{\prime}}=$ volume averages of the quantity $\phi$ on $\Omega, \Omega^{\prime}$, respectively.
$\langle\phi\rangle_{\text {eff }} \quad=$ particular volume average on $\Omega^{\prime}$.

\section{Introduction}

A double porosity medium is constituted of two porous systems of distinctly different porosity and permeabilities. This concept was introduced by Barenblatt $e t$ al (1960) for-the investigation of fissured porous media:-one of the two porous structures is associated with the fractures and the other one with the porous matrix. The behaviour of such a medium during fluid flow is of interest in many engineering fields (for instance, in petroleum engineering and in mining engineering). Consequently, it has been the subject of active research. The first investigations of double porosity media dealt with fissured reservoirs by means of phenomenological approaches (Barenblatt et al., 1960; Barenblat, 1963; Warren and Root, 1963). The basic assumption of this kind of model is that each point in space is associated with two pressures: the average fluid pressure in the fractures and the average fluid pressure in the micropores (Barenblatt et al., 1960). It is also assumed that the fluid is slightly compressible. In these models the laws are linear. The Barenblatt-Zheltov model (Barenblatt et al., 1960) shows an important characteristic of a double porosity system, the interporosity flow, i.e., the fluid exchange between the two constitutive media. As for the Warren and Root model (Warren and Root, 1963), it neglects the flow through the matrix block system; and the permeability of the matrix is usually considered as negligible compared to the fracture system permeability. This simplification is the subject of debate. In particular, let us note the works of Chen $(1989,1990)$, where it is claimed that it is necessary to solve the overall Barenblatt-Zheltov model, taking into account the flow through the matrix block system. These two theoretical models - the complete and the simplified model - have been taken up again by many authors to apply them to different domains of reservoir engineering. A state of the art in the knowledge of double porosity behaviour investigated with this kind of approach is well described by Gringarten (1984). For the particular case of slightly compressible fluids in double porosity systems, see Chen (1989).

On the other hand, the homogenization method (Bensoussan et al., 1978, Sanchez-Palencia, 1980) is also used for modelling double or single porosity media. In the case of sligthly compressible fluids, Arbogast et al. (1990) derive a double porosity model by means of the homogenization method, by considering Darcy's law at the microscopic level. To highlight the local effects at the pore and fracture scales, and the exchanges between the two porous structures, it is preferable to consider Navier-Stokes equations within the pore and the fracture systems. This is the case in Levy (1990), where the filtration of an incompressible 
fluid in fissured microporous rigid rock is investigated. One relationship between the separations of scales was investigated to show the influence of the fissure connectivity. As a result, the case of interest is when the pores and the fractures are connected. In other respects, compressible fluid flow through a deformable porous matrix with a single porosity is studied in a similar way in Auriault et al. (1990). It is shown there that there exists a macroscopic equivalent description only if the transient inertial term of the Navier-Stokes equations is negligible at the first order. Therefore, the behaviour is said to be quasi-static. Moreover, it is shown there that the richest macroscopic description is obtained for an $\mathrm{O}(1)$ macroscopic Strouhal number. This one is introduced by the mass balance equation. It is defined as the ration between the time derivative of the density and the mass flux.

Double porosity media introduce three separated scales, i.e., three scales with very different characteristic lengths: the pore scale, the fracture scale and the macroscopic scale. The method of asymptotic developments (i.e., the homogenization method) is very well adopted to this case; see Auriault and Boutin (1992, 1993, 1994), where it is applied to an incompressible fluid flow through a deformable porous matrix with double porosity, in the quasi-static and acoustic cases. The main result in these investigations is the strong dependence of the macroscopic behavior on the separations of scales. Here, we will take up again the same formalism and apply it in the context of highly compressible fluid flow through a rigid porous matrix.

The aim of the paper is to model very compressible fluid flow through double porosity media, and to compare the result to the two-pressure phenomenological models, in the case of slightly compressible fluid. In the second part of the paper, the physical domain of the study is defined. As in Auriault and Boutin (1992, 1993, 1994), we consider three cases of different relationships between the three separations of scales. The local equations that describe the flow and estimates required for homogenization are presented. Preliminary estimates of the order of magnitude of the fracture and pore velocities are then obtained, to simplify the following investigation. The three macroscopic behaviours are itemized in the third part of the paper. In each case, the macroscopic behaviour is described in the form of mass balance equations with Darcy's law. We show there the strong differences between the three cases, in particular, for the influence of the pore matrix. Depending on the separation of scales, it plays the role of a compressible fluid reservoir, or introduces memory effects, or is ignored, at the first order of approximation. The following reasoning and results are summarized in Auriault and Royer (1993b). In the fourth part, a form of the case II macroscopic description is compared to the Warren Root phenomenological approach. This kind of comparison was made in a previous paper (Auriault and Royer, 1993a), in the particular and simple case of the heat transfer in composites with double conductivity. By transferring these results to our problem, the pseudo-steady state phenomenological approaches are shown to give an inadequate model of the phenomenon. The homogenization method enables us to improve the model by introducing a new derivative term in the interporosity 


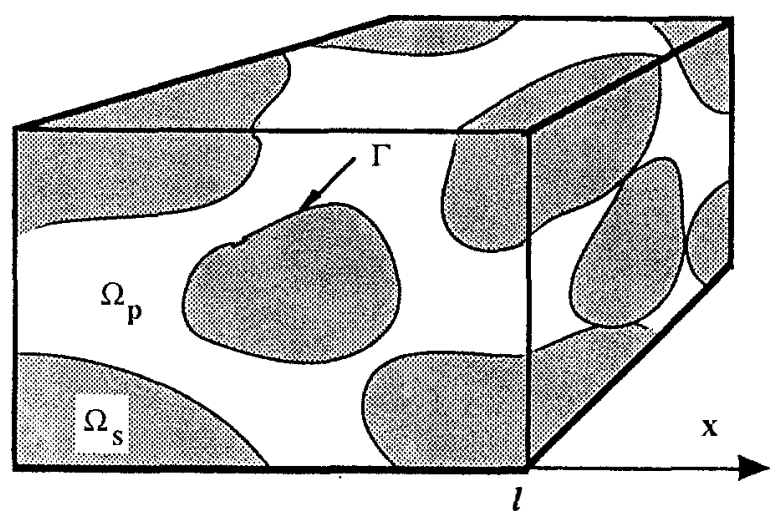

Fig. 1. Representation of the periodic cell at the pore scale. $\Omega_{\mathrm{p}}$ is the pore domain, $\Omega_{\mathrm{s}}$ the solid domain and $\Gamma$ their common boundary. $l$ is the microscopic characteristic length.

flow. Finally the Zimmerman et al. (1993) model is shown to be an approximation for the long-time behaviour of media with well-defined separations of scales (our case III).

\section{Problem Formulation}

\subsection{THE MEDIUM INVESTIGATED}

When there is a separation of scales, media with random or periodic thin structures lead to similar macroscopic behaviours (Auriault, 1991). Hence, without lost of generality, we assume the medium to be doubly periodic. On the pore scale, the medium is periodic with the space period $\Omega$ and the characteristic length is $l . \Omega_{\mathrm{s}}$ and $\Omega_{\mathrm{p}}$ are the domains occupied by the solid and the pores, respectively, and their common boundary is $\Gamma$ (Figure 1 ). On the fracture scale, a second porous structure exists of characteristic length $l^{\prime} \gg l$, periodic with the space period $\Omega^{\prime} . \Omega_{\mathrm{sp}}^{\prime}$ and $\Omega_{\mathrm{f}}^{\prime}$ are the domains occupied by the pore matrix block and the fractures, respectively, and their common boundary is $\Gamma^{\prime}$ (Figure 2). The pores and the fractures are assumed to be connected. Finally, $l^{\prime \prime} \gg l^{\prime} \gg l$ denotes the characteristic length of the macroscopic medium. The macroscopic behaviour appears to be strongly dependent upon the different separations of scales:

$$
\alpha=l / l^{\prime}, \quad \beta=l^{\prime} / l^{\prime \prime}, \quad \gamma=l / l^{\prime \prime} .
$$

As in Auriault and Boutin (1992, 1993, 1994), we will investigate the three following cases:

$$
\begin{array}{ll}
\text { case I } & : \beta=\mathrm{O}\left(\alpha^{2}\right)=\mathrm{O}\left(\varepsilon^{2}\right), \\
\text { case II } & : \alpha=\mathrm{O}(\beta)=\mathrm{O}(\varepsilon), \\
\text { case III } & : \alpha=\mathrm{O}\left(\beta^{2}\right)=\mathrm{O}\left(\varepsilon^{2}\right),
\end{array}
$$



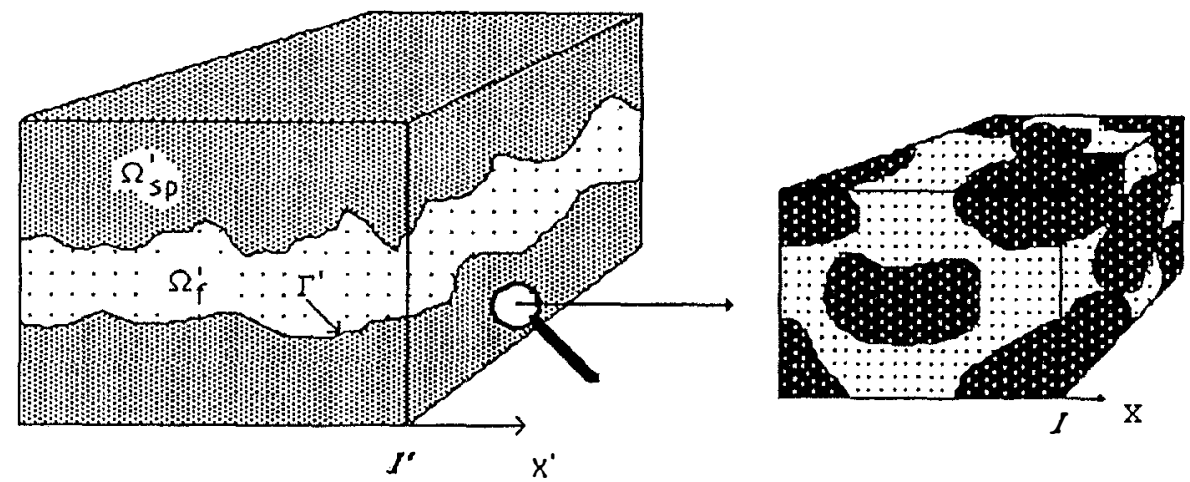

Fig. 2. Representation of the periodic cell at the fracture scale. $\Omega_{\mathrm{f}}^{\prime}$ is the pore domain, $\Omega_{\mathrm{sp}}^{\prime}$ the solid domain and $\Gamma$ their common boundary. $l^{\prime}$ is the microscopic characteristic length.

TABLE I. Three coal systems corresponding to cases I, II and III

\begin{tabular}{llll}
\hline & $l$ & $l^{\prime}$ & $l^{\prime \prime}$ \\
\hline Case I & $10^{-3} \mathrm{~m}$ & $2 \times 10^{-1} \mathrm{~m}$ & $10^{4} \mathrm{~m}$ \\
Case II & $10^{-3} \mathrm{~m}$ & $2 \times 10^{-1} \mathrm{~m}$ & $40 \mathrm{~m}$ \\
Case III & $5 \times 10^{-6} \mathrm{~m}$ & $2 \times 10^{-1} \mathrm{~m}$ & $40 \mathrm{~m}$ \\
\hline
\end{tabular}

where $\varepsilon \ll 1$.

For a reservoir, $l$ and $l^{\prime}$ are known. $l^{\prime \prime}$ is the macroscopic size, i.e., the seam coal size, the gas reservoir size, or the distance between gas wells, etc. Let us note that $l^{\prime \prime}$ can also be defined as the excitation characteristic length instead of coming from geometric consideration. Characteristic lengths for three different coal systems corresponding to the three cases are reported on Table I. An other example for a reservoir, taken from Van Golf-Racht (1982), is given Auriault and Boutin (1993).

\subsection{EQUATIONS}

For the sake of simplicity, we assume the system to be at rest at the initial time: velocities are zero-valued and pressure and density are supposed to be constant $\left(P_{0}\right.$ and $\rho_{0}$, respectively). We consider the perturbation of this equilibrium state. In the case of slow flow, we have the following equations, with $k=\mathrm{p}$ in the pores and $k=\mathrm{f}$ in the fractures.

\section{Momentum balance}

$\lambda$ and $\mu$ are viscosities, $\mathbf{v}_{k}$ is the velocity and $P_{k}$ denotes the increment of pressure. According to the previous assumptions, the flow is quasi-static and inertial terms are neglected. 


$$
\begin{aligned}
& \mu \Delta \mathbf{v}_{k}+(\lambda+\mu) \nabla\left(\nabla \cdot \mathbf{v}_{k}\right)-\nabla P_{k}=\left(\rho_{0}+\rho_{k}\right) \\
& \cdot\left[\frac{\partial \mathbf{v}_{k}}{\partial t}+\left(\mathbf{v}_{k} \cdot \nabla\right) \cdot \mathbf{v}_{k}\right]=\mathbf{0} .
\end{aligned}
$$

Mass balance

$$
\frac{\partial \rho_{k}}{\partial t}+\nabla \cdot\left(\left(\rho_{0}+\rho_{k}\right) \mathbf{v}_{k}\right)=0
$$

where $\rho_{0}$ is the initial density and $\rho_{k}$ the increment of density.

Gas state equation

For the sake of simplicity we assume the fluid to be linear. We consider the following incremental law:

$$
\rho_{k}=A P_{k} \quad \text { where } A \text { is a constant. }
$$

Boundary conditions

On $\Gamma$

$$
\mathbf{v}_{\mathrm{p} / \Gamma}=\mathbf{0}
$$

On $\Gamma^{\prime}$

$$
\mathbf{v}_{\mathrm{f} / \Gamma^{\prime}}=\left\langle\mathbf{v}_{\mathrm{p}}\right\rangle_{\Omega}=\frac{1}{|\Omega|} \int_{\Omega_{\mathrm{p}}} \mathbf{v}_{\mathrm{p}} \mathrm{d} \Omega .
$$

The condition (5) is demonstrated in Levy and Sanchez Palencia (1975). In particular, the volume average $\left\langle\mathbf{v}_{\mathrm{p}}\right\rangle_{\Omega}$ is shown to be equal to a surface average. Therefore it is a flux.

$$
P_{\mathrm{f}}=P_{\mathrm{p}}
$$

Initial conditions

$$
\begin{aligned}
& \mathbf{v}_{k}(t=0)=\mathbf{0} \\
& \rho_{k}(t=0)=0 \\
& P_{k}(t=0)=0
\end{aligned}
$$

2.3. PRELIMINARY STUDY OF THE ORDER OF MAGNITUDE OF THE VELOCITIES The aim of this section is to determine the relative order of magnitude between $\mathbf{v}_{\mathrm{p}}$ and $\mathbf{v}_{\mathrm{f}}$. As the ratio $v_{\mathrm{p}} / v_{\mathrm{f}}$ can be measured in $\varepsilon$ power, we put

$$
v_{\mathrm{p}} / v_{\mathrm{f}}=\mathrm{O}\left(\varepsilon^{h}\right), \quad \text { where } h \text { is a positive integer. }
$$


The gas is forced through the porous medium by a time-dependent difference of the pressure at the boundary of the macroscopic sample. When inertial forces are negligible, then the pressure term is balanced by the viscous term in the momentum balance

$$
\frac{\delta^{\prime \prime} P}{l^{\prime \prime}}=\mathrm{O}\left(\mu \frac{v_{\mathrm{p}}^{\mathrm{p}}}{l^{2}}\right)=\mathrm{O}\left(\mu \frac{v_{\mathrm{f}}^{\mathrm{p}}}{l^{2}}\right),
$$

where $\delta^{\prime \prime} P$ is a pressure difference applied to the macroscopic boundary and $v_{k}^{\mathrm{p}}$ is the velocity due to the macroscopic pressure gradient. Hence, we have

$$
v_{\mathrm{p}}^{\mathrm{p}} / v_{\mathrm{f}}^{\mathrm{p}}=\mathrm{O}\left(\alpha^{2}\right) .
$$

Now, according to the mass balance Equation (2), the medium is also subjected to temporal changes of pressure and so

$$
A \frac{\partial P}{\partial t}=\mathrm{O}\left(\rho \frac{v_{\mathrm{p}}^{t}}{l}\right)=\mathrm{O}\left(\rho \frac{v_{\mathrm{f}}^{t}}{l^{\prime}}\right),
$$

where $v_{k}^{t}$ denotes the velocity due to the temporal change of pressure. Hence, we deduce

$$
v_{\mathrm{p}}^{t} / v_{\mathrm{f}}^{t}=\mathrm{O}(\alpha)
$$

Now, the study of $\mathbf{v}_{\mathbf{f}}$ is made by means of developments in powers of the small parameter $\beta=l^{\prime} / l^{\prime \prime}$, which measures the separation of scale between the fractures and the macroscopic level. Therefore, the boundary condition (5) on $\Gamma^{\prime}$ imposes

$$
v_{\mathrm{p}} / v_{\mathrm{f}}=\mathrm{O}\left(\beta^{m}\right), \quad \text { where } m \text { is a positive integer. }
$$

Finally, according to Auriault et al. (1990), the homogenizability leads to:

$$
v_{\mathrm{f}}=\mathrm{O}\left(v_{\mathrm{f}}^{\mathrm{p}}\right)=\mathrm{O}\left(v_{\mathrm{f}}^{t}\right) .
$$

Therefore, we deduce from (8), (10), (12):

$$
v_{\mathrm{p}}^{\mathrm{p}} / v_{\mathrm{p}}^{t}=\mathrm{O}(\alpha) \text {. }
$$

Equation (13) means that the pore velocity due to the temporal change of pressure is always much greater than the velocity due to the macroscopic pressure gradient. Let us now consider the problem without the fractures. The pore velocity has then to be studied in the form of a development in powers of $\gamma=l / l^{\prime \prime}$, which measures the separation of scales between the pores and the macroscopic level. Equation (7) can be written in the form

$$
v_{\mathrm{p}}^{\mathrm{p}}=\mathrm{O}(\gamma)\left(\frac{\delta^{\prime \prime} P l^{2}}{l^{\prime \prime} \mu}\right)
$$


A quantity $\phi$ is said to be $\mathrm{O}_{\gamma}(\psi)$ if

$$
\gamma^{1 / 2} \leqslant \frac{\phi}{\psi} \leqslant \gamma^{-(1 / 2)}
$$

Adding the fractures does not change (14), since

$$
\delta^{\prime \prime} P / l^{\prime \prime}=\delta^{\prime} P / l^{\prime} .
$$

Consequently, the pore velocity will be $v_{\mathrm{p}}^{t}$ if, according to (13) and (14)

$$
\alpha=\mathrm{O}_{\gamma}(1),
$$

and if the condition (11) is checked, too. This one can be translated as follows:

$$
\alpha=\beta^{m}, \quad \text { where } m \text { is a positive integer. }
$$

Therefore, we deduce the following rule:

$$
\begin{aligned}
& v_{\mathrm{p}} / v_{\mathrm{f}}=\mathrm{O}\left(\varepsilon^{h}\right), \quad \text { where the positive integer } h \text { is defined as follows } \\
& \quad-\text { if } \alpha=\mathrm{O}_{\gamma}(1) \text { and if } \alpha=\beta^{m}: \varepsilon^{h}=\alpha, \\
& \text { - otherwise }: \varepsilon^{h}=\varepsilon \alpha .
\end{aligned}
$$

Hence, we obtain the following results in the three investigated cases:

In case I:

$$
\begin{aligned}
& \alpha=\varepsilon, \quad \beta=\varepsilon^{2}, \quad \gamma=\varepsilon^{3}, \\
& \alpha=\mathrm{O}_{\gamma}(1), \quad \text { but } \alpha \neq \beta^{m}, \\
& \Rightarrow v_{\mathrm{p}} / v_{\mathrm{f}}=\mathrm{O}(\alpha \varepsilon)=\mathrm{O}\left(\varepsilon^{2}\right) .
\end{aligned}
$$

In case II:

$$
\begin{aligned}
& \alpha=\varepsilon, \quad \beta=\varepsilon, \quad \gamma=\varepsilon^{2}, \\
& \alpha=\mathrm{O}_{\gamma}(1), \quad \alpha=\beta^{m}, \\
& \Rightarrow v_{\mathrm{p}} / v_{\mathrm{f}}=\mathrm{O}(\alpha)=\mathrm{O}(\varepsilon) .
\end{aligned}
$$

In case III:

$$
\begin{aligned}
& \alpha=\varepsilon, \quad \beta=\varepsilon, \quad \gamma=\varepsilon^{3}, \\
& \alpha \neq \mathrm{O}_{\gamma}(1), \\
& \Rightarrow v_{\mathrm{p}} / v_{\mathrm{f}}=\mathrm{O}(\alpha \varepsilon)=\mathrm{O}\left(\varepsilon^{3}\right) .
\end{aligned}
$$




\subsection{SPACE VARIABLES}

The three characteristic lengths allow us to introduce the three following dimensionless variables:

$$
\frac{\mathbf{x}^{\prime \prime}}{l}, \quad \frac{\mathbf{x}^{\prime \prime}}{l^{\prime}}, \quad \frac{\mathbf{x}^{\prime \prime}}{l^{\prime \prime}},
$$

which describe the pore, the fracture, and the macroscopic media, respectively. Each physical quantity is a function of these three variables. We use preferably three physical variables, which are as follows:

$$
\begin{array}{ll}
\mathbf{x}=\mathrm{O}\left(\gamma^{-1}\right) \mathbf{x}^{\prime \prime} & \text { for the pore medium, } \\
\mathbf{x}^{\prime}=\mathrm{O}\left(\beta^{-1}\right) \mathbf{x}^{\prime \prime} & \text { for the fracture medium, } \\
\mathbf{x}^{\prime \prime} & \text { for the macroscopic medium. }
\end{array}
$$

\subsection{TIME SCALES AND TIME VARIABLES}

The separation of space scales yields a separation of time scales (Auriault and Boutin, 1992, 1993, 1994). To highlight them, let us consider two fictitious reference displacement fields, $u_{\mathrm{p}}$ and $u_{\mathrm{f}}$, for the pores and the fractures, respectively, and let us assume that:

$$
u_{\mathrm{p}} / u_{\mathrm{f}}=\mathrm{O}(1)
$$

Then, we define the characteristic times, $T_{\mathrm{p}}$ and $T_{\mathrm{f}}$, by:

$$
\mathbf{v}_{\mathrm{p}}=\mathbf{u}_{\mathrm{p}} / T_{\mathrm{p}}, \quad \mathbf{v}_{\mathrm{f}}=\mathbf{u}_{\mathrm{f}} / T_{\mathrm{f}},
$$

from which:

$$
T_{\mathrm{f}} / T_{\mathrm{p}}=\mathrm{O}\left(\frac{v_{\mathrm{p}}}{v_{\mathrm{f}}}\right)=\mathrm{O}\left(\varepsilon^{h}\right) .
$$

From the time variable two dimensionless time variables can be defined:

$t / T_{\mathrm{f}}$ for the fractures, $\quad t / T_{\mathrm{p}}$ for the pores.

Because of the separation, each quantity is a function of these two times, too. We would sooner use the following variables:

$t$ for the fractured medium, $\quad T=\mathrm{O}\left(\varepsilon^{h}\right) t$ for the porous medium.

Hence, we obtain:

In case I: $\quad T_{\mathrm{f}} / T_{\mathrm{p}}=\mathrm{O}\left(\varepsilon^{2}\right)$, 
In case II: $T_{\mathrm{f}} / T_{\mathrm{p}}=\mathrm{O}(\varepsilon)$,

In case III: $T_{\mathrm{f}} / T_{\mathrm{p}}=\mathrm{O}\left(\varepsilon^{3}\right)$,

The previous choices determine an essential role of the variables $x^{\prime \prime}$ and $t$; we have adopted a macroscopic point of view (Auriault, 1991) for the medium description. Consequently, dimensionless numbers will be estimated by means of the characteristic length $l^{\prime \prime}$ and the characteristic time $T_{\mathrm{f}}$.

\subsection{DIMENSIONLESS NUMBERS}

The momentum balance (1) introduces the following dimensionless number:

$$
Q_{\mathrm{k}}=\frac{\left|\nabla P_{k}\right|}{\left|\mu \Delta \mathbf{v}_{k}\right|}
$$

$Q_{\mathrm{k}}$ has to be estimated as follows:

$$
Q_{\mathrm{k}}=\frac{\delta^{\prime \prime} P / l^{\prime \prime}}{\mu\left(u_{\mathrm{k}} / T_{\mathrm{f}} l^{\prime \prime 2}\right)}
$$

Now, according to (7) and (22), we have

$$
\mu \frac{u_{\mathrm{k}}}{T_{\mathrm{f}} l^{\prime 2}}=\mathrm{O}\left(\frac{\delta^{\prime \prime} P}{l^{\prime \prime}}\right) \text {. }
$$

Therefore:

$$
\mu \frac{u_{\mathrm{k}}}{T_{\mathrm{f}} l^{\prime \prime 2}}=\mathrm{O}\left(\mu \frac{u_{\mathrm{k}}}{T_{\mathrm{f}} l^{\prime 2}} \beta^{2}\right)=\mathrm{O}\left(\frac{\delta^{\prime \prime} P}{l^{\prime \prime}} \beta^{2}\right) .
$$

Hence:

$$
Q=Q_{\mathrm{f}}=Q_{\mathrm{p}}=\mathrm{O}\left(\beta^{-2}\right) .
$$

The mass balance equation (2) shows the Strouhal number

$$
S_{k}=\frac{\left|\partial \rho_{\mathrm{k}} / \partial t\right|}{\left|\nabla \cdot\left(\left(\rho_{0}+\rho_{k}\right) \mathbf{v}_{k}\right)\right|} .
$$

According to the results of Auriault et al. (1990), we investigate the case where

$$
S_{\mathrm{f}}=\mathrm{O}(1) \text {, }
$$


and we find

$$
S_{\mathrm{p}}=\mathrm{O}\left(\beta \varepsilon^{-h}\right) .
$$

Taking into account these estimations, the flow equations are written in the dimensionless normalized form, as follows:

$$
\begin{aligned}
& \beta^{2} \mu \Delta \mathbf{v}_{\mathrm{k}}+\beta^{2}(\lambda+\mu) \nabla\left(\nabla \cdot \mathbf{v}_{\mathrm{k}}\right)-\nabla P_{k}=\mathbf{0}, \\
& \frac{\partial \rho_{\mathrm{f}}}{\partial t}+\nabla \cdot\left(\left(\rho_{0}+\rho_{\mathrm{f}}\right) \mathbf{v}_{\mathrm{f}}\right)=0 \quad \text { in } \Omega_{\mathrm{f}}^{\prime}, \\
& \frac{\partial \rho_{\mathrm{p}}}{\partial t}+\beta^{-1} \varepsilon^{\mathrm{h}} \nabla \cdot\left(\left(\rho_{0}+\rho_{\mathrm{p}}\right) \mathbf{v}_{\mathrm{p}}\right)=0 \quad \text { in } \Omega_{\mathrm{p}}, \\
& \rho_{k}=A P_{k} \\
& \mathbf{v}_{\mathrm{p} / \Gamma}=\mathbf{0}, \\
& \mathbf{v}_{\mathrm{f} / \Gamma^{\prime}}=\left\langle\mathbf{v}_{\mathrm{p}}\right\rangle_{\Omega}, \\
& P_{\mathrm{f}}=P_{\mathrm{p}} \quad \text { on } \Gamma^{\prime} .
\end{aligned}
$$

\subsection{HOMOGENIZATION}

To perform the upscaling from the pore and the fracture scales to the macroscopic scale, we use a homogenization method. Taking advantage of the small parameter $\varepsilon$, the velocity, the density and the pressure fields are classically looked for in the form of asymptotic expansions. In the pore domain, the pressure and the density are of the order of magnitude $\mathrm{O}(1)$. By taking into account the above estimations, the pore velocity development may start with a $\varepsilon^{h}$ term. In the fracture domain all fields are of the order $\mathrm{O}(1)$. Hence:

$$
\begin{aligned}
& \phi_{\mathrm{p}}=\phi_{\mathbf{p}}^{0}\left(\mathbf{x}, \mathbf{x}^{\prime}, \mathbf{x}^{\prime \prime}, t, T\right)+\varepsilon \phi_{\mathrm{p}}^{1}\left(\mathbf{x}, \mathbf{x}^{\prime} \mathbf{x}^{\prime \prime}, t, T\right)+\cdots, \quad \phi=P, \rho, \\
& \mathbf{v}_{\mathrm{p}}=\varepsilon^{h} \mathbf{v}_{\mathrm{p}}^{h}\left(\mathbf{x}, \mathbf{x}^{\prime}, x^{\prime \prime}, t, T\right)+\varepsilon^{h+1} \mathbf{v}_{\mathrm{p}}^{h+1}\left(\mathbf{x}, \mathbf{x}^{\prime} \mathbf{x}^{\prime \prime}, t, T\right)+\cdots, \\
& \phi_{\mathrm{f}}=\phi_{\mathrm{f}}^{0}\left(\mathbf{x}^{\prime}, \mathbf{x}^{\prime \prime}, t, T\right)+\varepsilon^{r} \phi_{\mathrm{f}}^{r}\left(\mathbf{x}^{\prime} \mathbf{x}^{\prime \prime}, t, T\right)+\varepsilon^{2 r} \phi_{\mathrm{f}}^{2 r}\left(\mathbf{x}^{\prime}, \mathbf{x}^{\prime \prime}, t, T\right)+\cdots, \\
& \beta=\varepsilon^{r}, \quad \phi=\mathbf{v}, P, \rho .
\end{aligned}
$$

We now introduce the expansions (33) in the dimensionless equation set (27-32) taking into account that:

$$
\begin{aligned}
& \nabla_{x_{\imath}^{\prime \prime}} \text { is written }: \nabla_{x_{i}^{\prime \prime}}+\beta^{-1} \nabla_{x_{\imath}^{\prime}}+\gamma^{-1} \nabla_{x_{i}}, \\
& \frac{\partial}{\partial t} \text { becomes }: \frac{\partial}{\partial t}+\varepsilon^{h} \frac{\partial}{\partial T} .
\end{aligned}
$$




\section{The Macroscopic Models}

After introduction of the developments in the dimensionless equations, successive boundary value problems are obtained at the different orders of $\varepsilon$. For the case I, the detailed calculus is presented in a previous paper (Royer and Auriault, 1992). For the case II, the calculus is itemized in the Appendix. The three distinct derived macroscopic behaviours are written as follows:

\section{Case I}

$$
\left[n^{\prime}+\left(1-n^{\prime}\right) n\right] \frac{\partial P^{0}}{\partial t}-\nabla_{x^{\prime \prime}} \cdot\left(\left(P_{0}+P^{0}\right) \tilde{K}_{\mathrm{f}}\left(\mathbf{x}^{\prime \prime}\right) \nabla_{x^{\prime \prime}} P^{0}\right)=0,
$$

where $\quad \tilde{K}_{\mathrm{f}}$ is the fracture permeability,

$P_{0}$ is the intial pressure such as: $\rho_{0}=A P_{0}$,

$P^{0}=P_{\mathrm{p}}^{0}=P_{\mathrm{f}}^{0}$ is independent of the local space variables on $\Omega_{\mathrm{sp}}^{\prime}$ and $\Omega_{\mathrm{f}}^{\prime}$,

$n=\left|\Omega_{p}\right| /\left|\Omega_{\mathrm{sp}}^{\prime}\right|$ and $n^{\prime}=\left|\Omega_{f}^{\prime}\right| /\left|\Omega^{\prime}\right|$ are the porosities in the pores and in the fractures respectively.

\section{Case II}

$$
n^{\prime} \frac{\partial P_{\mathrm{f}}^{0}}{\partial t}+n \frac{\left\langle P_{\mathrm{p}}^{0}\right\rangle_{\mathrm{eff}}}{\partial t}-\nabla_{x^{\prime \prime}} \cdot\left[\left(P_{0}+P_{\mathrm{f}}^{0}\right) \tilde{K}_{\mathrm{f}}\left(\mathbf{x}^{\prime \prime}\right) \nabla_{x^{\prime \prime}} P_{\mathrm{f}}^{0}\right]=0,
$$

where $\left\langle P_{\mathrm{p}}^{0}\right\rangle_{\text {eff }}=1 /\left|\Omega^{\prime}\right| \int_{\Omega_{\mathrm{sp}}^{\prime}} P_{\mathrm{p}}^{0} \mathrm{~d} \Omega$,

$P_{\mathrm{f}}^{0}$ and $\rho_{\mathrm{f}}^{0}$ are independent of the local space variable on $\Omega_{\mathrm{f}}^{\prime}$,

$P_{\mathrm{p}}^{0}$ and $\rho_{\mathrm{p}}^{0}$ are independent of the local space variable on $\Omega_{\mathrm{p}}$,

$P_{\mathrm{p}}^{0}$ is given by the set:

$$
\begin{aligned}
& n\left(\partial P_{\mathrm{p}}^{0} / \partial t\right)-\nabla_{x^{\prime}} \cdot\left[P_{\mathrm{p}}^{0} \tilde{K}_{\mathrm{p}}\left(\mathbf{x}^{\prime}\right) \nabla_{x^{\prime}} P_{\mathrm{p}}^{0}\right]=0, \\
& P_{\mathrm{p}}^{0}=\rho_{\mathrm{f}}^{0} \quad \text { on } \Gamma^{\prime}
\end{aligned}
$$

and some given initial condition $P_{\mathrm{p}}^{0}\left(\mathbf{x}^{\prime}, 0\right)$.

$\tilde{K}_{\mathrm{p}}$ is the pore permeability.

The solution of this set can be put in the form:

$$
P_{\mathrm{p}}^{0}=\mathcal{F}\left(P_{\mathrm{f}}^{0}\right),
$$

where $\mathcal{F}$ is a nonlinear time-dependent functional exhibiting memory effect.

\section{Case III}

$$
n^{\prime} \frac{\partial P_{\mathrm{f}}^{0}}{\partial t}-\nabla_{x^{\prime \prime}} \cdot\left[\left(P_{0}+P_{\mathrm{f}}^{0}\right) \tilde{K}_{\mathrm{f}}\left(\mathbf{x}^{\prime \prime}\right) \nabla_{x^{\prime \prime}} P_{\mathrm{f}}^{0}\right]=0 .
$$


In case I, the macroscopic behaviour (34) is a nonlinear mass balance equation, with the classical Darcy's law for the fractures. It resembles single porous medium behaviour. The peculiarity of this behaviour lies in the source term $\left[n^{\prime}+(1-\right.$ $\left.\left.n^{\prime}\right) n\right]\left(\partial P^{0} / \partial t\right)$, where appears the pore matrix influence. At the first order of approximation, the pore flow is taken into account through this term, only. The pore matrix plays the role of a compressible fluid flow reservoir.

In case II, the flow through the pores strongly influences the macroscopic behaviour: it imposes memory effects and added strong nonlinearities appear at the macroscopic scale. This is the case of the greatest interest, which shows how important the local effects can be.

Finally, in case III, the pores are entirely ignored. It is exactly the result that would be obtained by a straightforward homogenization from the fracture scale to the macroscopic level. In this case, the medium can be considered as a single porosity system, as the first order of approximation.

The nonlinearities in the three models take their origin in the high compressibility of the fluid. They disappear with lower compressibility (see Section 4.4).

It is interesting to introduce characteristic times $\tau_{\mathrm{p}}$ and $\tau_{\mathrm{f}}$ for the changes in the pore and the fracture flows, respectively

$$
v_{\mathrm{p}}=l^{\prime} / \tau_{\mathrm{p}}, \quad v_{\mathrm{f}}=l^{\prime \prime} / \tau_{\mathrm{f}}, \quad \tau_{\mathrm{f}} / \tau_{\mathrm{p}}=\mathrm{O}\left(\beta^{-1} \varepsilon^{h}\right) .
$$

In cases I and II, $\tau_{\mathrm{f}} / \tau_{\mathrm{p}}=\mathrm{O}(1)$. Changes in the fracture and in the pore flows occur at the same time, as can be seen from (34) and (35), under fully transient conditions. As $t$ goes to infinity, case II yields a pseudo-steady-state behaviour (Warren and Root, 1963).

In case III, $\tau_{\mathrm{f}} / \tau_{\mathrm{p}}=\mathrm{O}\left(\varepsilon^{2}\right)$. For short times $\mathrm{O}\left(\tau_{\mathrm{f}}\right)$, as shown by (36), the flow occurs in the fractures, only. For longer times $\mathrm{O}\left(\tau_{\mathrm{p}}\right)$, flow appears in the pores and the fractures practically work as perfect drains, at nearly constant pressure. The flow in the porous matrix occurs under fully transient conditions. The Zimmerman et al. model (1993), is an approximation of the long-time behaviour in case III. As $t$ goes to infinity, the flows becomes pseudo-static, as in case II.

\section{Comparison with the Phenomenological Pseudo-Steady State Models}

A rigorous phenomenological model for highly compressible fluid flow in a double porosity medium is not available in the literature, to our knowledge. The existing models, for example in Barenblatt et al. (1990), are unreliable because of the seepage law the authors are using: as demonstrated in Auriault et al. (1990), the seepage law for a compressible fluid is the Darcy law. The rigorous existing models, mainly the Barenblatt model (Barenblatt et al. 1960) and the Warren and Root model (Warren and Root, 1963), assume that the fluid is slightly compressible. Moreover, it is assumed that the interporosity flow $q$, i.e., the flux of fluid from 
matrix to fractures, occurs in response to the fracture-pore difference of pressure. This is assumed to describe a pseudo-steady state flow

$$
q=s K_{\mathrm{p}}\left(P_{\mathrm{p}}-P_{\mathrm{f}}\right)
$$

where $s$ is a characteristic coefficient of the fractured rock proportional to the specific surface of the block.

We first introduce these models. Then we compare them to the case II model when $t$ goes to infinity, but in a linearized form and in the case of a slightly compressible fluid. This comparison will allow us to improve the interporosity term.

\subsection{THE COMPLETE BARENBLATT MODEL}

To describe a double porosity model, Barenblatt et al. (1960) introduce two pressure fields at each point of space: $P_{\mathrm{f}}$ and $P_{\mathrm{p}}$ for the fractures and the pores, respectively. The model applies to the case of a slightly deformable matrix and a slightly compressible fluid. When the porous matrix is rigid, the model reduces to the two following equations, with our definitions for the permeabilities

$$
\begin{aligned}
& K_{\mathrm{f}} \Delta P_{\mathrm{f}}=n^{\prime} C^{*} \frac{\partial P_{\mathrm{f}}}{\partial t}-s K_{\mathrm{p}}\left(P_{\mathrm{p}}-P_{\mathrm{f}}\right), \\
& K_{\mathrm{p}} \Delta P_{\mathrm{p}}=n C^{*} \frac{\partial P_{\mathrm{p}}}{\partial t}+s K_{\mathrm{p}}\left(P_{\mathrm{p}}-P_{\mathrm{f}}\right),
\end{aligned}
$$

where $C^{*}$ is the gas compressibility coefficient. The permeability tensors are assumed to be isotropic: $\tilde{K}_{\mathrm{f}}=K_{\mathrm{f}} \tilde{I}, \tilde{K}_{\mathrm{p}}=K_{\mathrm{p}} \tilde{I}$, where $\tilde{I}$ is the identity tensor.

\subsection{THE SIMPLIFIED BARENBLATT MODEL}

In the case of a fractured porous medium, Barenblatt theory neglects the fracture porosity $n^{\prime}$ and the fluid flow in the porous matrix. Consequently, the pore permeability takes only place to describe interporosity flow, i.e., the fluid exchange between the pore matrix and the fractures

$$
\begin{aligned}
& K_{\mathrm{f}} \Delta P_{\mathrm{f}}+s K_{\mathrm{p}}\left(P_{\mathrm{p}}-P_{\mathrm{f}}\right)=0, \\
& n C^{*} \frac{\partial P_{\mathrm{p}}}{\partial t}+s K_{\mathrm{p}}\left(P_{\mathrm{p}}-P_{\mathrm{f}}\right)=0,
\end{aligned}
$$

Elimination of $P_{\mathrm{p}}$ in (39) and (40) yields

$$
\frac{\partial P_{\mathrm{f}}}{\partial t}-\frac{K_{\mathrm{f}}}{s K_{\mathrm{p}}} \frac{\partial}{\partial t}\left(\Delta P_{\mathrm{f}}\right)-\frac{K_{\mathrm{f}}}{n C^{*}} \Delta P_{\mathrm{f}}=0 .
$$




\subsection{THE WARREN AND ROOT MODEL}

The double porosity model of Warren and Root is also a simplification of the complete Barenblatt model. The porosity and the compressibility coefficient in the fractures are not neglected, but the fluid flow in the pore matrix is neglected in this model

$$
\begin{aligned}
& K_{\mathrm{f}} \Delta P_{\mathrm{f}}=n^{\prime} C^{*} \frac{\partial P_{\mathrm{f}}}{\partial t}-s K_{\mathrm{p}}\left(P_{\mathrm{p}}-P_{\mathrm{f}}\right), \\
& n C^{*} \frac{\partial P_{\mathrm{p}}}{\partial t}+s K_{\mathrm{p}}\left(P_{\mathrm{p}}-P_{\mathrm{f}}\right)=0,
\end{aligned}
$$

In Fourier space, the system is written as

$$
\begin{aligned}
& K_{\mathrm{f}} \Delta P_{\mathrm{f}}=i \omega n^{\prime} C^{*} P_{\mathrm{f}}-s K_{\mathrm{p}}\left(P_{\mathrm{P}}-P_{\mathrm{f}}\right), \\
& i \omega n C^{*} P_{\mathrm{p}}+s K_{\mathrm{p}}\left(P_{\mathrm{P}}-P_{\mathrm{f}}\right)=0,
\end{aligned}
$$

where $\omega$ is the pulsation.

Elimination of $P_{\mathrm{p}}$ between (44) and (45) yields

$$
K_{\mathrm{f}} \Delta P_{\mathrm{f}}=i \omega n^{\prime} C^{*} P_{\mathrm{f}}+\frac{i \omega n C^{*} s K_{\mathrm{p}}}{s K_{\mathrm{p}}+i \omega n C^{*}} P_{\mathrm{f}} .
$$

\subsection{THE LINEARIZED MODEL OBTAINED BY HOMOGENIZATION}

As shown in Section 3, the pseudo-steady-state corresponds to the long-time behaviour in case II. For the comparison to be possible, the case II model is simplified to slightly compressible fluids. We put

$$
\rho_{0}+\rho_{k}=\rho_{0}\left(1+C P_{k}\right), \quad k=\mathrm{p}, \mathrm{f},
$$

where: $\quad\left|C P_{k}\right| \ll 1 ; C$ is the compressibility coefficient. We assume the permeabilities to be constant and isotropic. The model reduces to

$$
n^{\prime} C^{*} \frac{\partial P_{\mathrm{f}}^{0}}{\partial t}+n G^{*} \frac{\partial\left\langle P_{\mathrm{p}}^{0}\right\rangle_{\mathrm{eff}}}{\partial t}-K_{\mathrm{f}} \Delta_{x^{\prime \prime}} P_{\mathrm{f}}^{0}=0, \quad C^{*}=C / \rho_{0}
$$

$P_{\mathrm{p}}^{0}$ is the solution of the following diffusive equation

$$
K_{\mathrm{p}} \Delta_{x^{\prime}} P_{\mathrm{p}}^{0}=n C^{*} \frac{\partial P_{\mathrm{p}}^{0}}{\partial t},
$$

with the boundary condition

$$
P_{\mathrm{p}}^{0}=P_{\mathrm{f}}^{0} \quad \text { on } \Gamma^{\prime} .
$$


Proceeding by Fourier analysis, we have then to solve

$$
K_{\mathrm{p}} \Delta_{x^{\prime}} P_{\mathrm{p}}^{0}=n C^{*} i \omega P_{\mathrm{p}}^{0}, \quad P_{\mathrm{p}}^{0}=P_{\mathrm{f}}^{0} \quad \text { on } \Gamma^{\prime} .
$$

Let us define $W$ by

$$
P_{\mathrm{p}}^{0}=P_{\mathrm{f}}^{0}+W \text {. }
$$

The boundary-value problem becomes

$$
K_{\mathrm{p}} \Delta_{x^{\prime}} W=n C^{*} i \omega\left(P_{\mathrm{f}}^{0}+W\right), \quad W=0 \quad \text { on } \Gamma^{\prime} .
$$

The solution is linear with respect to $P_{\mathrm{f}}^{0}$

$$
W\left(\mathbf{x}^{\prime}, \mathbf{x}^{\prime \prime}, \omega\right)=-k\left(\mathbf{x}^{\prime}, \mathbf{x}^{\prime \prime}, \omega\right) P_{\mathrm{f}}^{0},
$$

where $k$ is complex and $\omega$ dependent and represents the solution for $P_{\mathrm{f}}^{0}=-1$. Equation (48) can then be written as follows in Fourier space:

$$
\begin{aligned}
& K_{\mathrm{f}} \Delta_{x^{\prime \prime}} P_{\mathrm{f}}^{0}=\left[n^{\prime} C^{*}+n\left(1-n^{\prime}\right) C^{*}-n C^{*}\langle k\rangle_{\text {eff }}\right] i \omega P_{\mathrm{f}}^{0}, \\
& \langle k\rangle_{\text {eff }}=\frac{1}{\left|\Omega^{\prime}\right|} \int_{\Omega_{\mathrm{sp}}^{\prime}} k \mathrm{~d} \Omega .
\end{aligned}
$$

Taking the inverse Fourier transform of (49) we obtain the description for a transient excitation

$$
\begin{aligned}
& K_{\mathrm{f}} \Delta_{x^{\prime \prime}} P_{\mathrm{f}}^{0}=(\left.n^{\prime} C^{*}+n\left(1-n^{\prime}\right) C^{*}\right) \frac{\partial P_{\mathrm{f}}^{0}}{\partial t} \\
&-n C^{*} \int_{-\infty}^{t} \hat{K}(t-\tau) \frac{\partial^{2} P_{\mathrm{f}}^{0}}{\partial t^{2}} \mathrm{~d} \tau,
\end{aligned}
$$

where $\hat{K}(t)$ is the inverse Fourier transform of $\langle k\rangle_{\text {eff }} / \mathrm{i} \omega$ and characterizes the memory effects induced by the double porosity structure of the medium. The memory function $\hat{K}(t)$ is similar to the memory function introduced in Auriault (1983) and used again in Auriault and Royer (1993a), for transient heat transfer in double conductivity composites. In the general case $\hat{K}(t)$ is a sum of exponential terms. An equivalent formulation is obtained by integrating by parts the integral in equation (50), noticing that all time derivatives of $\hat{K}(t)$ are vanishing when $t$ goes to infinity:

$$
\begin{aligned}
K_{\mathrm{f}} \Delta_{x^{\prime \prime}} P_{\mathrm{f}}^{0}=( & \left.n^{\prime} C^{*}+n\left(1-n^{\prime}\right) C^{*}\right) \frac{\partial P_{\mathrm{f}}^{0}}{\partial t}-d_{2} \frac{\partial^{2} P_{\mathrm{f}}^{0}}{\partial t^{2}} \\
& -d_{3} \frac{\partial^{3} P_{\mathrm{f}}^{0}}{\partial t^{3}}-\cdots-d_{n} \frac{\partial^{n} P_{\mathrm{f}}^{0}}{\partial t^{n}}-\cdots
\end{aligned}
$$

The memory of the past is replaced by the knowledge of all time derivatives at the present time. The convolution product represents the interporosity flow. It introduces strong difficulties in numerical investigations. Linear memory effects were also introduced by Bibby (1981) to describe the mass transport of solutes in dual-porosity media. 
For simplicity, we limit the comparison to the Warren and Root model. An analogous investigation is conducted in Auriault and Royer (1993a) for heat transfer in composites with double conductivities. The reader is referred to this paper for details. We need a long time approximation of the linearized homogenization result of (50). The memory function $\hat{K}$ can be represented by an infinite sum of exponential terms:

$$
\hat{K}(t)=\sum_{p=1}^{\infty} a_{p} \mathrm{e}^{-b_{p} t}, \quad b_{1}<b_{2}<\cdots
$$

The long time behaviour can therefore be approximated by

$$
\hat{K}(t) \approx a_{1} \mathrm{e}^{-b_{1} t} .
$$

Introducing this value into (49) gives

$$
K_{\mathrm{f}} \Delta_{x^{\prime \prime}} P_{\mathrm{f}}^{0}=C^{*}\left[n^{\prime}+n\left(1-n^{\prime}\right)-n \frac{a_{1} i \omega}{b_{1}+i \omega}\right] i \omega P_{\mathrm{f}}^{0} .
$$

Identifying with the Warren and Root model (46) yields the impossible identity

$$
\frac{n C^{*} s K_{\mathrm{p}}}{s K_{\mathrm{p}}+n C^{*} i \omega} \equiv n C^{*}\left(1-n^{\prime}-\frac{a_{1} i \omega}{b_{1}+i \omega}\right) .
$$

The approximation (52) can be used to improve the phenomenological model (see Auriault and Royer, 1993a). It gives the interporosity flow in the form

$$
q=s K_{\mathrm{p}}\left(P_{\mathrm{p}}+\theta \frac{\partial P_{\mathrm{p}}}{\partial t}-P_{\mathrm{f}}\right)
$$

where $\theta$ is a constant.

\subsection{THE ZIMMERMAN et al. MODEL}

Many authors have attempted to improve the interporosity flow term to obtain a larger time-scale range of validity. The model introduced by Zimmerman et al. (1993) concerns slightly compressible fluids. To approximate the interporosity flow, the authors assume that the pressure in the fractures changes abruptly at $t=0$ and remains positive at all posivite times. They also replace the memory function by an ad hoc approximation. As a result, the linear memory term is replaced by a nonlinear expression - but more convenient for numerical purposes - with respect 
to the pressures in the pores and the fractures. Clearly, such a model will be valid if the characteristic times are such as:

$$
\tau_{\mathrm{f}} \ll \tau_{\mathrm{p}} .
$$

Hence, the Zimmerman model cannot be compared to cases I and II. This model corresponds to case III, where $\tau_{\mathrm{f}} / \tau_{\mathrm{p}}=\mathrm{O}\left(\varepsilon^{2}\right)$, for times of the order of magnitude of $\tau_{\mathrm{p}}$.

At such times, the pressure in the fractures is nearly a constant $P_{0}$. The flow in the pores occurs under fully transient conditions, with a memory function similar to the memory function $\hat{K}(t)$ in case II. This is exactly the situation described by Zimmerman et al. Finally, the abrupt change of pressure in the fractures appears as an unnecessary assumption. The Zimmerman et al. model applies also to case II with fully transient flows in the fractures and the pores.

\section{Conclusion}

We have investigated the macroscopic behaviour of compressible fluid flow through a rigid porous medium with double porosity. This behaviour is strongly dependent upon the relative value of the separations of scales: in the three cases of interest, very different macroscopic behaviours are obtained. This was already shown in an other context in earlier papers using the same methodology (Auriault and Boutin, 1992, 1993, 1994). As in those papers, the largest coupled effects between the pores and the fractures are obtained in the case II, i.e., for equal separation of scales. The fluid compressibility introduces strong nonlinear memory effects. For a slightly compressible fluid, pseudo-steady-state phenomenological approaches were shown to be a very rough approximation in the case II, when $t$ goes to infinity. The phenomenological models were improved by the introduction of new time derivative terms in the interporosity flow. Finally, the Zimmerman et al. model was shown to belong to the case III. The investigation of porous media with several porosities is of the greatest importance for many domains of applications, for instance, in petroleum engineering or in mining engineering. The latter concerns in particular the study of the coal-gas outbursts, to determine precisely the setting of factors that contribute to the occurrence of an outburst (Strzelecki et al., 1993). This phenomenon, which impedes coal mining, is the subject of intensive experimental and theoretic research. Unless coal-gas outbursts depend on other phenomena such as diffusion or surface adsorption and the related solid swelling, the multi-porosity structure of the coal plays an important role. In this sense, the above results can be considered as a first step in the modelling.

\section{Appendix: Case II - Homogenization}

In case II, we have: 


$$
\begin{aligned}
& \alpha=\mathrm{O}(\beta)=\mathrm{O}(\varepsilon), \quad \varepsilon \ll 1, \\
& \alpha=\frac{l}{l^{\prime}}, \quad \beta=\frac{l^{\prime}}{l^{\prime \prime}}, \quad \gamma=\frac{l}{l^{\prime \prime}} .
\end{aligned}
$$

Therefore, the space and time variables are written as

$$
\begin{aligned}
& \mathbf{x}=\mathrm{O}\left(\varepsilon^{-2}\right) \mathbf{x}^{\prime \prime} \quad \mathbf{x}^{\prime}=\mathrm{O}\left(\varepsilon^{-1}\right) \mathbf{x}^{\prime \prime} \quad \mathbf{x}^{\prime \prime}, \\
& t, \quad T=\varepsilon t .
\end{aligned}
$$

The dimensionless numbers have the following orders of magnitude from Section 2.6:

$$
Q=\mathrm{O}\left(\varepsilon^{-2}\right), \quad S=\mathrm{O}(1) .
$$

Finally, the dimensionless equations are as follows:

$$
\begin{aligned}
& \varepsilon^{2} \mu \Delta \mathbf{v}_{k}+\varepsilon^{2}(\lambda+\mu) \nabla\left(\nabla \cdot \mathbf{v}_{k}\right)-\nabla P_{k}=\mathbf{0}, \\
& \frac{\partial \rho_{k}}{\partial t}+\nabla \cdot\left(\left(\rho_{0}+\rho_{k}\right) \mathbf{v}_{k}\right)=0, \\
& \rho_{k}=A P_{k}, \\
& \mathbf{v}_{\mathrm{p} / \Gamma}=0, \\
& \mathbf{v}_{\mathrm{f} / \Gamma^{\prime}}=\left\langle\mathbf{v}_{\mathrm{p}}\right\rangle^{\Omega}, \\
& P_{\mathrm{f}}=P_{\mathrm{p}} \text { on } \Gamma^{\prime} .
\end{aligned}
$$

According to the study of the orders of magnitude, Section 2.3, it follows that

$$
\frac{v_{\mathrm{p}}}{v_{\mathrm{f}}}=\mathrm{O}(\varepsilon) .
$$

We must then consider the different fields in the forms

$$
\begin{aligned}
& \phi_{\mathrm{p}}=\phi_{\mathrm{p}}^{0}\left(\mathbf{x}, \mathbf{x}^{\prime}, \mathbf{x}^{\prime \prime}, t, T\right)+\varepsilon \phi_{\mathrm{p}}^{1}\left(\mathbf{x}, \mathbf{x}^{\prime}, \mathbf{x}^{\prime \prime}, t, T\right)+\cdots, \quad \phi=P, \rho, \\
& \mathbf{v}_{\mathrm{p}}=\varepsilon \mathbf{v}_{\mathrm{p}}^{1}\left(\mathbf{x}, \mathbf{x}^{\prime}, \mathbf{x}^{\prime \prime}, t, T\right)+\varepsilon^{2} \mathbf{v}_{\mathrm{p}}^{2}\left(\mathbf{x}, \mathbf{x}^{\prime}, \mathbf{x}^{\prime \prime}, t, T\right)+\cdots, \\
& \phi_{\mathrm{f}}=\phi_{\mathrm{f}}^{0}\left(\mathbf{x}^{\prime}, \mathbf{x}^{\prime \prime}, t, T\right)+\varepsilon \phi_{\mathbf{f}}^{1}\left(\mathbf{x}^{\prime}, \mathbf{x}^{\prime \prime}, t, T\right)+\varepsilon^{2} \phi_{\mathrm{f}}^{2}\left(\mathbf{x}^{\prime}, \mathbf{x}^{\prime \prime}, t, T\right)+\cdots, \\
& \phi=\mathbf{v}, P, \rho .
\end{aligned}
$$

For the gradient and the temporal derivative, we must take into account that

$$
\begin{aligned}
& \nabla_{x_{\imath}^{\prime \prime}} \text { is written }: \nabla_{x_{\imath}^{\prime \prime}}+\varepsilon^{-1} \nabla_{x_{\imath}^{\prime}}+\varepsilon^{-2} \nabla_{x_{i}}, \\
& \frac{\partial}{\partial t} \text { becomes }: \frac{\partial}{\partial t}+\varepsilon \frac{\partial}{\partial T} .
\end{aligned}
$$

We have now to analyze the boundary-value problem introduced at the different orders of $\varepsilon$, in the pores and in the fractures. 


\section{A.1. THE PORE SYSTEM}

The pressure field at the first order:

Equation (A1) at the order of $\varepsilon^{-2}$ and Equation (A3) at the order of $\varepsilon^{0}$ yield

$$
\begin{aligned}
& \nabla_{x} P_{\mathrm{p}}^{0}=\mathbf{0}, \\
& P_{\mathrm{p}}^{0}=A \rho_{\mathrm{p}}^{0} .
\end{aligned}
$$

From Equations (A7) and (A8) we deduce

$$
\begin{aligned}
& P_{\mathrm{p}}^{0}=P_{\mathrm{p}}^{0}\left(\mathbf{x}^{\prime}, \mathbf{x}^{\prime \prime}, t, T\right), \\
& \rho_{\mathrm{p}}^{0}=\rho_{\mathrm{p}}^{0}\left(\mathbf{x}^{\prime}, \mathbf{x}^{\prime \prime}, t, T\right) .
\end{aligned}
$$

The pore filtration velocity at the first order:

Equation (A1) at the order of $\varepsilon^{1}$, Equation (A2) at the order of $\varepsilon^{-1}$ and Equation (A4) at the order of $\varepsilon^{1}$ yield

$$
\begin{aligned}
& \nu \Delta_{x} \mathbf{v}_{\mathrm{p}}^{1}+(\lambda+\mu) \nabla_{x}\left(\nabla_{x} \cdot \mathbf{v}_{\mathrm{p}}^{1}\right)-\nabla_{x}^{\prime} P_{\mathrm{p}}^{0}-\nabla_{x} P_{\mathrm{p}}^{1}=\mathbf{0}, \\
& \nabla_{x} \cdot \mathbf{v}_{\mathrm{p}}^{1}=0, \\
& \mathbf{v}_{\mathrm{p} / \Gamma}^{1}=\mathbf{0},
\end{aligned}
$$

where $\mathbf{v}_{\mathrm{p}}^{1}$ and $P_{\mathrm{p}}^{1}$ and $\Omega$-periodic.

This linear boundary-value problem, of variable $x$, determines

$$
\begin{aligned}
& \mathbf{v}_{\mathrm{p}}^{1}=-\tilde{k}_{\mathrm{p}}(\mathbf{x}) \nabla_{x^{\prime}} P_{\mathrm{p}}^{0}, \\
& P_{\mathrm{p}}^{1}=\tau_{\mathrm{p}}(\mathbf{x}) \nabla_{x^{\prime}} P_{\mathrm{p}}^{0}+\bar{P}_{\mathrm{p}}^{1}\left(\mathbf{x}^{\prime}, \mathbf{x}^{\prime \prime}, t, T\right) .
\end{aligned}
$$

The pore mass balance:

Equation (A2) at the order of $\varepsilon^{0}$ yields

$$
\frac{\partial \rho_{\mathrm{p}}^{0}}{\partial t}+\nabla_{x^{\prime}} \cdot\left(\left(\rho_{0}+\rho_{\mathrm{p}}^{0}\right) \mathbf{v}_{\mathrm{p}}^{1}\right)+\nabla_{x}\left(\left(\rho_{0}+\rho_{\mathrm{p}}^{0}\right) \mathbf{v}_{\mathrm{p}}^{2}+\rho_{\mathrm{p}}^{1} \mathbf{v}_{\mathrm{p}}^{1}\right)=0 .
$$

By integration on $\Omega_{\mathrm{p}}$, Equation (A16) leads to the following compatibility condition:

$$
\left\langle\frac{\partial \rho_{\mathrm{p}}^{0}}{\partial t}+\nabla_{x^{\prime}} \cdot\left(\left(\rho_{0}+\rho_{\mathrm{p}}^{0}\right) \mathbf{v}_{\mathrm{p}}^{1}\right)\right\rangle_{\Omega}=0
$$


We deduce then the behaviour in

$\Omega_{\mathrm{sp}}^{\prime}$

$$
n \frac{\partial \rho_{\mathrm{p}}^{0}}{\partial t}-\nabla_{x^{\prime}} \cdot\left[\left(\rho_{0}+\rho_{\mathrm{p}}^{0}\right)\left(\tilde{K}_{\mathrm{p}} \nabla_{x^{\prime}} P_{\mathrm{p}}^{0}\right)\right]=0
$$

or

$$
n \frac{\partial P_{\mathrm{p}}^{0}}{\partial t}-\nabla_{x^{\prime}} \cdot\left[\left(P_{0}+P_{\mathrm{p}}^{0}\right)\left(\tilde{K}_{\mathrm{p}} \nabla_{x^{\prime}} P_{\mathrm{p}}^{0}\right)\right]=0
$$

where

$$
n=\frac{\left|\Omega_{\mathrm{p}}\right|}{\left|\Omega_{\mathrm{sp}}^{\prime}\right|} \text { is the pore domain porosity, }
$$

and

$\tilde{K}_{\mathrm{p}}=\left\langle\tilde{k}_{\mathrm{p}}\right\rangle_{\Omega}$ is the pore permeability.

\section{A.2. THE FRACTURE SYSTEM}

The pressure field at the first order:

Equation (A1) at the order of $\varepsilon^{-1}$ and Equation (A3) at the order of $\varepsilon^{0}$ yield

$$
\begin{aligned}
& \nabla_{x^{\prime}} P_{\mathrm{f}}^{0}=\mathbf{0}, \\
& P_{\mathrm{f}}^{0}=A \rho_{\mathrm{f}}^{0} .
\end{aligned}
$$

From (A20) and (A21), we deduce:

$$
\begin{aligned}
& P_{\mathrm{f}}^{0}=P_{\mathrm{f}}^{0}\left(\mathbf{x}^{\prime \prime}, t, T\right), \\
& \rho_{\mathrm{f}}^{0}=\rho_{\mathrm{f}}^{0}\left(\mathbf{x}^{\prime \prime}, t, T\right) .
\end{aligned}
$$

The fracture filtration velocity at the first order:

Equation (A1) at the order of $\varepsilon^{0}$, Equation (A2) at the order of $\varepsilon^{-1}$ and Equation (A5) at the order of $\varepsilon^{0}$ yield

$$
\begin{aligned}
& \mu \Delta_{x^{\prime}} \mathbf{v}_{\mathbf{f}}^{0}+(\lambda+\mu) \nabla_{x^{\prime}}\left(\nabla_{x^{\prime}} \cdot \mathbf{v}_{\mathbf{f}}^{0}\right)-\nabla_{x^{\prime \prime}} P_{\mathrm{f}}^{0}-\nabla_{x^{\prime}} P_{\mathrm{f}}^{1}=\mathbf{0}, \\
& \nabla_{x^{\prime}} \cdot \mathbf{v}_{\mathrm{f}}^{0}=\mathbf{0} \\
& \mathbf{v}_{\mathrm{f} / \Gamma^{\prime}}^{0}=\left\langle\mathbf{v}_{\mathrm{p}}^{0}\right\rangle_{\Omega}=\mathbf{0},
\end{aligned}
$$

where $\mathbf{v}_{\mathrm{f}}^{0}$ and $P_{\mathrm{f}}^{1}$ are $\Omega^{\prime}$-periodic. 
This classical boundary-value problem determines

$$
\begin{aligned}
& \mathbf{v}_{\mathrm{f}}^{0}=-\tilde{k}_{\mathrm{f}}\left(\mathbf{x}^{\prime}\right) \nabla_{x^{\prime \prime}} P_{\mathrm{f}}^{0}, \\
& P_{\mathrm{f}}^{1}=\tau_{\mathrm{f}}\left(\mathbf{x}^{\prime}\right) \nabla_{x^{\prime \prime}} P_{\mathrm{f}}^{0}+\bar{P}_{\mathrm{f}}^{1}\left(\mathbf{x}^{\prime \prime}, t, T\right) .
\end{aligned}
$$

The macroscopic mass balance:

Equation (A2) at the order of $\varepsilon^{0}$ yields

$$
\frac{\partial \rho_{\mathrm{f}}^{0}}{\partial t}+\nabla_{x^{\prime \prime}} \cdot\left(\left(\rho_{0}+\rho_{\mathrm{f}}^{0}\right) \mathbf{v}_{\mathrm{f}}^{0}\right)+\nabla_{x^{\prime}} \cdot\left(\left(\rho_{0}+\rho_{\mathrm{f}}^{0}\right) \mathbf{v}_{\mathrm{f}}^{1}+\rho_{\mathrm{f}}^{1} \mathbf{v}_{\mathrm{f}}^{0}\right)=0 .
$$

By integration on $\Omega^{\prime}$, we obtain

$$
n^{\prime} \frac{\partial \rho_{\mathrm{f}}^{0}}{\partial t}+\nabla_{x^{\prime \prime}} \cdot\left(\left(\rho_{0}+\rho_{\mathrm{f}}^{0}\right)\left\langle\mathbf{v}_{\mathrm{f}}^{0}\right\rangle_{\Omega^{\prime}}\right)+\left\langle\nabla_{\mathbf{x}^{\prime}} \cdot\left(\left(\rho_{0}+\rho_{\mathrm{f}}^{0}\right) \mathbf{v}_{\mathrm{f}}^{1}\right)\right\rangle_{\Omega^{\prime}}=0
$$

where

$$
n^{\prime}=\frac{\left|\Omega_{\mathrm{f}}^{\prime}\right|}{\left|\Omega^{\prime}\right|} \text { is the fracture domain porosity, }
$$

Now, according to the boundary condition,

$$
\left\langle\nabla_{x^{\prime}} \cdot\left(\left(\rho_{0}+\rho_{\mathrm{f}}^{0}\right) \mathbf{v}_{\mathrm{f}}^{1}\right)\right\rangle_{\Omega^{\prime}}=\frac{\rho_{0}+\rho_{\mathrm{f}}^{0}}{\left|\Omega^{\prime}\right|} \int_{\Gamma}\left\langle\mathbf{v}_{\mathrm{p}}^{1}\right\rangle_{\Omega} \cdot \mathbf{n}^{\prime} \mathrm{d} S .
$$

Integrating equation (A18) on $\Omega_{\mathrm{sp}}^{\prime}$, and taking into account that:

$$
P_{\mathrm{f}}^{0}=P_{\mathrm{p}}^{0} \quad \text { on } \Gamma^{\prime},
$$

we obtain

$$
\int_{\Gamma}\left\langle\mathbf{v}_{\mathrm{p}}^{1}\right\rangle_{\Omega} \cdot \mathbf{n}^{\prime} \mathrm{d} S=\frac{1}{\rho_{0}+\rho_{\mathrm{f}}^{0}} n \frac{\partial}{\partial t} \int_{\Omega_{\mathrm{sp}}^{\prime}} \rho_{\mathrm{p}}^{0} \mathrm{~d} \Omega
$$

and therefore

$$
\left\langle\nabla_{x^{\prime}} \cdot\left(\left(\rho_{0}+\rho_{\mathrm{f}}^{0}\right) \mathbf{v}_{\mathrm{f}}^{1}\right)\right\rangle_{\Omega^{\prime}}=n \frac{\partial\left\langle\rho_{\mathrm{p}}^{0}\right\rangle_{\mathrm{eff}}}{\partial t},
$$

where

$$
\left\langle\rho_{\mathrm{p}}^{0}\right\rangle_{\mathrm{eff}}=\frac{1}{\left|\Omega^{\prime}\right|} \int_{\Omega_{\mathrm{sp}}^{\prime}} \rho_{\mathrm{p}}^{0} \mathrm{~d} \Omega .
$$


Equation (A30) also becomes:

$$
n^{\prime} \frac{\partial \rho_{\mathrm{f}}^{0}}{\partial t}+n \frac{\left\langle\rho_{\mathrm{p}}^{0}\right\rangle_{\mathrm{eff}}}{\partial t}-\nabla_{x^{\prime \prime}} \cdot\left[\left(\rho_{0}+\rho_{\mathrm{f}}^{0}\right) \tilde{K}_{\mathrm{f}}\left(\mathbf{x}^{\prime \prime}\right) \nabla_{x^{\prime \prime}} P_{\mathrm{f}}^{0}\right]=0
$$

Finally, the flow is described as follows:

$$
n^{\prime} \frac{\partial P_{\mathrm{f}}^{0}}{\partial t}+n \frac{\left\langle P_{\mathrm{p}}^{0}\right\rangle_{\mathrm{eff}}}{\partial t}-\nabla_{x^{\prime \prime}} \cdot\left[\left(P_{0}+P_{\mathrm{f}}^{0}\right) \tilde{K}_{\mathrm{f}}\left(\mathbf{x}^{\prime \prime}\right) \nabla_{x^{\prime \prime}} P_{\mathrm{f}}^{0}\right]=0
$$

where $\quad \tilde{K}_{\mathrm{f}}$ is the fracture permeability,

$$
\left\langle P_{\mathrm{p}}^{0}\right\rangle_{\mathrm{eff}}=\frac{1}{\left|\Omega^{\prime}\right|} \int_{\Omega_{\mathrm{sp}}^{\prime}} P_{\mathrm{p}}^{0} \mathrm{~d} \Omega \text {. }
$$

$P_{\mathrm{p}}^{0}$ is given by the following boundary value problem:

$$
\begin{aligned}
& n \frac{\partial P_{\mathrm{p}}^{0}}{\partial t}-\nabla_{x^{\prime}} \cdot\left[P_{\mathrm{p}}^{0} \tilde{K}_{\mathrm{p}}\left(\mathbf{x}^{\prime}\right) \nabla_{x^{\prime}} P_{\mathrm{p}}^{0}\right]=0, \\
& P_{\mathbf{f}}^{0}=P_{\mathbf{p}}^{0} \quad \text { on } \Gamma^{\prime} .
\end{aligned}
$$

It gives:

$P_{\mathrm{p}}^{0}=\mathcal{F}\left(P_{\mathrm{f}}^{0}\right)$, where $\mathcal{F}$ is a nonlinear time-dependent functional exhibiting memory effect.

\section{Acknowledgement}

This work was sponsored by the Polish Scientific Research Committee in Warsaw (Grant KBN No. 7S10304305).

\section{References}

Arbogast, T., Douglas, J., and Hornung, U.: 1990, Derivation of the double porosity of single-phase flow via homogenization theory, SIAM J. Math. Anal. 21(4), 823-836.

Auriault, J. L.: 1983, Effective macroscopic description for heat conduction in periodic composites, J. Heat Mass Transfer, 26(6), 861-869.

Auriault, J. L.: 1991, Heterogeneous medium. Is an Equivalent macroscopic description possible? Int. J. Eng. Sci 29(7), 785-795.

Auriault, J. L. and Boutin, C.:1992, Deformable porous Media with double porosity. Quasi-statics I: coupling effects, Transport in Porous Media 7, 63-82.

Auriault, J. L. and Boutin, C.: 1993, Deformable porous media with double porosity. Quasi-statics II: memory effects, Transport in Porous Media 10, 153-169.

Auriault, J. L. and Boutin, C.: 1994, Deformable porous media with double porosity. III: Acoustics, Transport in Porous Media 14, 143-162.

Auriault, J. L. and Royer, P.: 1993a, Double conductivity media: a comparison between phenomenological and homogenization approaches, Int. J. Heat Mass Transfer 36(10), 2613-2621. 
Auriault, J. L. and Royer, P.: 1993b, Ecoulement d'un gaz dans un milieu poreux à double porosité, C. R. Acad. Sci. Paris ser 2 (II) 431-436.

Auriault, J. L., Strzelecki, T., Bauer, J., and He, S.:1990, Porous deformable media saturated by a very compressible fluids: Quasi-statics Eur. J. Mech. A/Solids 9(4), 373-392.

Barenblatt, G. I.: 1963, On certain boundary value problems for the equations of seepage of liquid in fissured rocks, $P M M, 27(2), 348-350$.

Barenblatt, G. I., Zheltov, Iu., and Kochina, I. N.: 1960, Basic concept in the theory of seepage of homogeneous liquids in fissured rocks, $P M M, 24(5), 852-864$.

Barenblatt, G. I., Entov, V. M., and Ryzhik, V. M.:1990, Theory of Fluid Flows through Natural Rocks, Kluwer Academic Publishers, Dordrecht.

Bensoussan, A., Lions J. L., and Papanicolaou G. C.: 1978, Asymptotic Analysis for Periodic Structures, North-Holland Publishing Co., Amsterdam.

Bibby, R.: 1981, Mass transport of solutes in dual-porosity media, Water Resour: Res. 1075-1081.

Chen. Z. X.: 1989, Transient flow of slightly compressible fluids through double-porosity, doublepermeability systems - A state of the art review, Transport in Porous Media 4, 147-184.

Chen, Z. X.: 1990, Analytical solutions for the double-porosity, double-permeability and layered systems, J. Petrol. Sci. Eng. 5, 1-24.

Gringarten A. C.: 1984, Interpretation of tests in fissured and multilayered reservoirs with double porosity behavior: theory and practice, J. Petrol. Technol. Distinguished Authors Series 4, 549464.

Hornung, U. and Showalter, R. E.: 1990, Diffusion models for fractured media, J. Math. Anal. Appl. 147, 69-80.

Levy, T.: 1990, Filtration in a porous fissured rock. Influence of the fissure connexity, Eur. J. Mech. B/Fluids 9(4), 309-327.

Levy T. and Sanchez-Palencia, E.: 1975, On boundary conditions for fluid flow in porous media, Int. J. Engng. Sci. 13, 923-940.

Royer, P. and Auriault, J. L.: 1992, Very compressible fluid flow through a porous rigid medium with double porosity, Studia Geot. Mech. 13(1-2), 65-77.

Strzelecki, T., Bauer, J., and Auriault, J. L.: 1993, Constitutive equations of a gas-filled two phase medium, Transport in Porous Media 10, 197-202.

Sanchez-Palencia, E.: 1990, Non homogeneous media and vibration theory, Lecture Notes in Physics 127, Springer, Berlin.

Van Golf-Racht, T. D.:1982, Fundamentals of Fractured Reservoir Engineering, Elsevier, New York.

Warren, J. O. and Root, P. J.: 1963, The behaviour of naturally fractured reservoirs, Soc. Petrol. Eng. J., 254-255.

Zimmerman, R. W., Chen, G., Hadgu, T., and Bodvarsson, G. S.: 1993, A numerical dual-porosity model with semianalytical treatment of fracture/matrix flow, Water Resour. Res. 29(7), 2127 2137. 